\title{
The crystalline structure of the phenazine overlayer physisorbed on a graphite surface
}

Adam Y. Brewer ${ }^{\mathrm{a}}$, Marco Sacchi ${ }^{\mathrm{b}}$, Julia E. Parker ${ }^{\mathrm{c}}$, Chris Truscott $^{\mathrm{a}}$, Steve Jenkins ${ }^{\mathrm{b}}$ and Stuart M. Clarke ${ }^{\mathrm{a}, *}$

${ }^{a}$ Department of Chemistry and BP Institute, University of Cambridge, Cambridge, United

Kingdom; ${ }^{b}$ Department of Chemistry, University of Cambridge, Cambridge, United Kingdom;

${ }^{c}$ Diamond Light Source Limited, Diamond House, Harwell Science and Innovation Campus,

Didcot, United Kingdom

*Corresponding Author: Stuart M. Clarke, BP Institute, Bullard Laboratories, Madingley Road, Cambridge, United Kingdom, CB3 0EZ; stuart@bpi.cam.ac.uk 


\section{The crystalline structure of the phenazine overlayer physisorbed on a graphite surface}

The monolayer crystal structure of phenazine adsorbed on graphite is determined by a combination of synchrotron X-ray diffraction and DFT calculations. The molecules adopt a rectangular unit cell with lattice parameters $a=13.55 \AA$ and $b=10.55 \AA$, which contains 2 molecules. The plane group of the unit cell is $p 2 g g$, and each molecule is essentially flat to the plane of the surface, with only a small amount of out-of-plane tilt. Density Functional Theory (DFT) calculations find a minimum energy structure with a unit cell which agrees within $7.5 \%$ with that deduced by diffraction. DFT including dispersion force corrections $(\mathrm{DFT}+\mathrm{D})$ calculations help to identify the nature of the intermolecular bonding. The overlayer interactions are principally van der Waals, with a smaller contribution from weak $\mathrm{C}-\mathrm{H} \cdots \mathrm{N}$ hydrogen bonds. This behaviour is compared with that of 4,4'-bipyridyl.

Keywords: monolayer, physisorption, synchrotron, DFT, phenazine

\section{Introduction}

The formation of non-covalent interactions in physisorbed monolayers is of continuing interest. There has been much work addressing the role of hydrogen bonding in controlling molecular self-assembly. Molecular physisorbed overlayers that have strong hydrogen bonds include alcohols [1-7], fatty acids [8-12] and amides [13, 14]. These strong, directional hydrogen bonds can overcome the inherent close packing that arises from the van der Waals (vdW) interactions leading to open molecular arrangements resembling chicken-wire supramolecular networks [15].

Recently, we have characterized the monolayer structures of 4,4'-bipyridyl (bpy) [16] and 1,2-bis(4-pyridyl)ethylene (bpe) [17] using synchrotron X-ray diffraction. These are illustrated in Figure 1 and are important species - with the overlayer crystal structures suggesting the formation of weak hydrogen bonds - as well as key components in halogen bonded molecular co-crystals [18]. In this work, we address the overlayer structure of phenazine on graphite at sub- 
monolayer coverage. The molecular structure of phenazine is also illustrated in Figure 1. All of these species are polyheterocyclic nitrogen compounds, but with different spatial arrangements of the rings. As with bpy and bpe, our interest in phenazine stems primarily from its ability to form strong and robust supramolecular interactions. However, the overlayer structure of pure phenazine is interesting in its own right, as well as being important for rationalising the interactions of phenazine with other molecules in the overlayer.

Here, we present the overlayer X-ray diffraction pattern for phenazine and the calculated overlayer crystal structure based on the experimental diffraction pattern. This is then compared to the results of DFT calculations of the same species and contrasted with similar calculations for 4,4'-bipyridyl.

[Figure 1 near here]

\section{Methods}

\subsection{Experimental}

The experimental approaches employed to obtain the diffraction patterns from physisorbed overlayers using synchrotron X-rays has been detailed elsewhere [19]. In this work, the diffraction patterns were recorded on Beamline I1 1 at Diamond Light Source, UK [20]. The Xray wavelength used was $1.033787 \AA$ with a detector zero angle offset of $0.00803^{\circ}$ as determined by Rietveld refinement of a silicon standard (NIST SRM 640c).

The graphite substrate used was Papyex, an exfoliated recompressed graphite foil purchased from Le Carbon. Papyex is a compressed powder of graphite crystallites, where the crystallites have a preferred orientation arising from the compression process. This preferred 
orientation can be exploited to maximize the recorded intensity from the adsorbed overlayer by concentrating diffracted intensity onto the detector. The Papyex used was determined to have a surface area of $27.5 \mathrm{~m}^{2} \mathrm{~g}^{-1}$ by nitrogen adsorption.

Phenazine was purchased from Sigma Aldrich with a purity of $99.6 \%$ determined by gas liquid chromatography as stated on the certificate of analysis. It was used without further purification. Graphite (3.003 g) was dosed with phenazine (17.88 $\mathrm{mg}$ ) by placing both in a Pyrex glass tube. The tube was evacuated to a pressure of $c a .0 .1 \mathrm{mbar}$ and sealed under vacuum. Phenazine is a solid with a low vapour pressure at room temperature, so little of the adsorbate is expected to have been lost through this process. The sealed glass tube was heated to $480 \mathrm{~K}$ for 3 hours before being allowed to cool slowly to room temperature (over the course of $c a$. 10 hours). Finally, the tube was broken open and the dosed graphite recovered.

Dosed graphite was cut into $3 \mathrm{~mm}$ diameter discs and stacked into a glass capillary such that the scattering vector was in the plane of the preferentially ordered crystallites. The sample was rotated on the instrument to enhance powder averaging, and the diffraction pattern recorded simultaneously over the angular range $1^{\circ}$ to $90^{\circ}$ using a position sensitive detector [21]. The sample temperature was controlled with a cryostream (Oxford Cryostreams, UK).

The quantity of adsorbate required was initially estimated from the size and shape of the phenazine molecule and the specific surface area of the substrate; subsequent calculations based on the experimentally determined overlayer structure gave a coverage of 0.51 equivalent monolayers, within the sub-monolayer regime. Here we have defined a full equivalent monolayer in terms of the number of molecules required to cover the surface of the substrate (based on the specific surface area of the substrate and the area of each adsorbate molecule). 
Differential scanning calorimetry was performed using a Perkin Elmer Pyris 1 power compensation calorimeter, calibrated with indium (for temperature and enthalpy) and heptane (for temperature).

\subsection{Computational}

In this work we used the density functional theory (DFT) code CASTEP [22]. More details of the DFT calculations are described by Sacchi et al. [23, 24]. Intermolecular bonding contributions originating from long range dispersion forces are not included in the Hamiltonian formulation of DFT. Although new functionals that account for weak dipole/induced dipole and other vdW forces are currently being developed [25], the most common procedure for calculating the energy and structure of physisorbed systems is to use the standard correction methodology based on atomic pair contributions and Lennard-Jones potentials developed by Grimme [26]. In this work we applied the dispersion force correction methodology developed by Tkatchenko and Scheffler [27] in which the $C_{6}$ coefficients of the pair potential are calculated directly from the electron density of the system. The ground-state energy of the system is calculated with the PerdewBurke-Ernzerhof exchange-correlation functional [28]. The plane waves were expanded to a kinetic energy cut-off of $340 \mathrm{eV}$, the electronic energy was converged to $10^{-6} \mathrm{eV}$ and the force tolerance for the cell optimization was $0.05 \mathrm{eV} / \AA$. In the present calculations the substrate was not included in the model, and an unconstrained geometry optimisation starting from the experimental structures was used. 


\section{Results}

\subsection{Synchrotron X-ray Diffraction}

The overlayer diffraction pattern for phenazine at a coverage of 0.51 equivalent monolayers is shown in Figure 2. This pattern was recorded between 214 and 239 K. To prepare this figure, the small angle Porod scattering (which arises from the external shape of the graphite crystallites [29]) and graphite background have been subtracted. Hence, the remaining peaks in this pattern are due solely to diffraction from the physisorbed overlayer. Due to the small amount of material present in the overlayer, the intensity of scattering from the adsorbate is rather weak. Therefore, the data has been averaged over seven points to reduce noise. The "sawtooth" lineshape of the peaks is characteristic of diffraction from 2D layers, and confirms that phenazine does indeed form a solid, crystalline 2D overlayer at this temperature.

Diffraction patterns from this sample were recorded over the temperature range $120 \mathrm{~K}$ $300 \mathrm{~K}$. The peaks indicative of the solid overlayer disappear at $c a .260 \mathrm{~K}$, which implies that this is the temperature at which the overlayer melts. This compares to the bulk melting point of $c a$. $444 \mathrm{~K}$ [30]. Hence the overlayer melting point is 0.59 of the bulk melting point. This is typical of many physisorbed overlayers which often melt at temperatures below the bulk melting point when at sub-monolayer coverages. Previous experience suggests that there is a small thermal lag between the cryostream temperature and the sample temperature; hence, this temperature is taken as indicative of the melting point only. Differential scanning calorimetry measurements performed on the sample indicate a phase transition at $274 \mathrm{~K}$, which is taken as a more accurate melting point of the overlayer.

The diffraction data recorded in this experiment was analysed as described elsewhere [8]. Experimentally, data can only be recorded over a limited range of momentum transfer, Q. Due 
to the limited number of X-ray reflections available, the fit to the data must ideally be constrained as much as possible. Therefore, the structure of the phenazine molecule used in the fitting was based upon the "bulk" three dimensional crystal structure of the $\beta$-polymorph, and only rigid rotations and translations of this molecule were considered [31]. The bulk structure was determined by X-ray diffraction, which tends to underestimate the C-H bond lengths [32]. Because we are particularly interested in intermolecular distances in the overlayer, the C-H bond lengths determined by X-ray diffraction (approximately $0.96 \AA$ ) were lengthened to $1.075 \AA$ in our fitting procedure. This longer bond is based on DFT calculations [33], and is in agreement with typical C-H bond lengths found by neutron diffraction [32], which provides a more accurate measure of $\mathrm{C}-\mathrm{H}$ bond length.

In our structural solution, rectangular unit cells and high symmetry plane groups, in which the molecules have fewer degrees of freedom, are considered before lower symmetry plane groups. There are several analytical models for the lineshape of two-dimensional diffraction peaks. In this work, the Gaussian, Lorentzian and Lorentzian-squared lineshapes of Schildberg and Lauter were considered [34]. The Lorentzian-squared model agreed most closely with the experimental data, and so has been used for the final refinement. This model includes terms for the size and preferential orientation of the graphite crystallites. A single temperature factor set to unity was used.

The agreement between the experimental and calculated patterns can be quantified using the "goodness of fit" parameter, $R$, where

$$
R=\frac{\sum_{h, k}\left(I_{o b s}-I_{c a l c}\right)^{2}}{\sum_{h, k}\left(I_{o b s}\right)^{2}}
$$

and $I_{o b s}$ and $I_{\text {calc }}$ are the observed and calculated intensities at the Bragg positions. The reduced 
chi-squared, $\chi^{2}$ red, can also be used as a measure of the goodness of fit:

$$
\chi_{r e d}^{2}=\frac{1}{N-n-1} \sum \frac{\left(I_{o b s}-I_{c a l c}\right)^{2}}{\sigma^{2}}
$$

where $N$ is the number of data points and $n$ the number of fitted parameters. However, as discussed elsewhere, the values of these parameters are not directly comparable with those of conventional bulk diffraction patterns [17].

The experimental pattern was indexed with a rectangular unit cell of dimensions $a=$ 13.55(5) $\AA, b=10.55(5) \AA$ and $v=90^{\circ}$. It was found that a high symmetry structure with $p 2 g g$ plane group fitted the experimental diffraction pattern. The unit cell is large enough to accommodate two phenazine molecules lying flat on the surface. When flat on a surface, phenazine possesses a two-fold rotation axis, which coincides with the two-fold rotation axes of the unit cell in a $p 2 g g$ symmetry structure. This fixes the position of the molecules within the unit cell, so that the molecules are centred at the origin of the cell and at $(1 / 2,1 / 2)$. Because the two molecules are symmetry-related by the two glide lines, they cannot be independently rotated about the surface normal (the $z$-direction), but rather the rotation of the two molecules about their $z$-axis is coupled. Therefore, if the phenazine molecules are constrained to lie flat in a plane parallel to the graphite surface, then the only freely variable parameter is the rotation of the molecules about the surface normal. The best fit obtained using this model is shown in Figure 2(a). This fit has $R=0.051$ and $\chi_{\text {red }}^{2}=3.27$. The calculated pattern captures the main features of the experimental pattern very well, particularly given the highly constrained nature of the structural model.

[Figure 2 near here] 
The fit can be slightly improved by relaxing the constraint that the molecules must lie parallel to the underlying graphite substrate. The pattern is much more sensitive to changes in the $\mathrm{x}$ - and $\mathrm{y}$ - co-ordinates of the atoms, i.e. the in-plane structure. Therefore, as the molecules are rotated up from the surface, the change in the calculated pattern arises predominantly from the change in the projection of the atomic co-ordinates onto the plane rather than the change in structure normal to the surface per se. This means that the calculated pattern is relatively insensitive to small rotations out of the plane, where the $\mathrm{x}$ - and $\mathrm{y}$ - co-ordinates do not change significantly $\left(+/-5^{\circ}\right)$. This also means that it is only possible to infer the magnitude, but not the sense, of rotation from the experimental pattern.

Rotations about two (orthogonal) axes were considered: about the $\mathrm{N}-\mathrm{N}$ axis, and the long axis of the molecule (perpendicular to the N-N axis). It was found that the best fit resulted from a rotation of $\sim 15^{\circ}$ about the N-N axis with no rotation about the long axis. This fit has $R=0.031$ and $\chi_{\text {red }}^{2}=2.83$. The fit is shown in Figure 3 .

[Figure 3 near here]

Given that the overlayer is no longer flat, it does not strictly have $p 2 g g$ symmetry. Instead, the two-fold rotation axes become centres of inversion, and the glide axes become either glide planes or screw axes. The unit cell contains two phenazine molecules, and each molecule can be rotated in either direction about its N-N axis. This results in two possible structures that would fit the experimental data, differing in whether the second molecule has the same or opposite sense of rotation compared to the first molecule. If the second molecule has the same 
sense of rotation as the first, this results in a glide plane parallel to the b-axis, and a screw axis parallel to the a-axis; conversely, if the second molecule has the opposite rotation, there will be screw axis parallel to the b-axis and a glide plane parallel to the a-axis. We have named the structures based on the symmetry element parallel to the b-axis. Hence, we refer to the first case as "glide" and the second case as "screw". These two arrangements are illustrated in Figure 4.

[Figure 4 near here]

It is not possible to confirm whether the overlayer is commensurate with the graphite substrate from the X-ray data. However, if the overlayer lattice parameters are an integer multiple of the graphite lattice parameters, this is a strong indication that the overlayer is

commensurate with the substrate. The lattice parameter of the underlying graphite is $a_{g}=2.46 \AA$, which compares with the measured overlayer lattice parameter, $a=13.55(5) \AA$. The $a$-axis is commensurate, with $2 a=11 a_{g}$, if $a=13.53 \AA$. This is within the error of the experimental measurement, and so we conclude that the $a$-axis is commensurate. The overlayer $b$-axis then corresponds to the graphite $\sqrt{ } 3 a_{g}$ direction. For the $b$-axis to be commensurate, this would require $b=10.65 \AA$ (compared to the experimentally determined $b=10.55(5) \AA$ ). This larger value of $b$ would result in a noticeable shift of the peaks away from their experimental positions, and so the unit cell does not appear to be commensurate in the $b$-direction. However, we cannot exclude more complex long range relationships between the adsorbed layer and the underlying graphite.

\subsection{Computational Results}

Several DFT calculations from the phenazine overlayer have been performed including where the molecules are constrained to be flat or to have the same lattice parameters as the structure 
calculated by diffraction above. The lowest energy structure, where the structural parameters have been allowed to float, corresponds to essentially the same structure as that determined by diffraction $\left(\mathrm{a}=12.809 \AA, \mathrm{b}=9.775 \AA \mathrm{v}=89.74^{\circ}\right)$ although the lattice parameters are slightly shorter (approx 7.5\%). The molecules are tilted at approximately $16^{\circ}$, very simlar to that experimentally determined, and the 'glide' molecular arrangement is lower energy than the 'screw' arrangement. The 'screw' arrangement and the structure with the molecules parallel to the surface ('flat' arrangement) are, respectively, $0.049 \mathrm{eV}$ and $0.057 \mathrm{eV}$ less stable than the glide arrangement.

Without vdW interactions, the screw and glide arrangements are rather similar in energy, although these are still somewhat more stable than the flat structure. Interestingly, the lattice parameters are rather expanded and closer to the structure determined by diffraction (only $3 \%$ compressed).

It should be noted that the DFT calculations correspond to the zero temperature structures. With such low energy differences, it is likely that the experimental structure is a statistical average of these low energy structures at the experimental temperature of approximately $200 \mathrm{~K}$.

The total binding energy of the structure is $0.68 \mathrm{eV}$ per cell of which $0.46 \mathrm{eV}$ is due to $\mathrm{vdW}$ interactions, and the remaining $0.22 \mathrm{eV}$ is attributed to mainly weak $\mathrm{C}-\mathrm{H} \cdots \mathrm{N}$ Hydrogen bonding.

\subsubsection{4,4'-bipyridyl (bpy)}

It is interesting to compare the DFT simulations of phenazine with bpy. Figure 5 illustrates the electron density difference maps for bpy. 
[Figure 5 near here]

The lowest energy structure for the BPY overlayer determined by DFT+D calculations has a unit cell of $a=11.253 \AA, b=11.371 \AA$, $v=90.01^{\circ}$. This is in very good agreement with that determined by diffraction (within 0.7\%) [16]. In these calculations, the initial configurations of the molecules are flat and the two bpy molecules have been allowed to re-orientate. All the intramolecular bond lengths and angles are also unconstrained. The total interaction energy is $0.87 \mathrm{eV}$ per cell, consisting of $0.39 \mathrm{eV}$ from vdW interactions and $0.48 \mathrm{eV}$ from the weak $\mathrm{N} \cdots \mathrm{H}$ hydrogen bonds. (Recall phenazine: Total $0.68 \mathrm{eV}$; vdW $0.46 \mathrm{eV} ; 0.22 \mathrm{eV}$ weak H-bonding). Hence we conclude that the difference between these two molecules is essentially contained in the H-bonding contributions. This weaker hydrogen bonding in phenazine is also evident in the longer $\mathrm{N}-\mathrm{H}$ distance in the overlayer.

\section{Discussion}

The overlayer structures of phenazine determined by synchrotron X-ray diffraction and DFT calculations are in good agreement. Both suggest that the tilted structure of phenazine is favoured (approximately $0.06 \mathrm{eV}$ more stable than the flat structure). Without the inclusion of vdW forces in the DFT calculation, it was found that the tilted structure was still more stable, indicating that the tilt does not arise from vdW forces alone. As introducing a tilt lengthens the $\mathrm{H} \cdots \mathrm{N}$ distance $(0.13 \AA)$, it seems unlikely that the tilt arises from the optimization of nontraditional H-bonds. Instead, we postulate that a possible cause of the tilt is to minimize the repulsion between the $\pi$-orbitals of neighbouring molecules. Whilst it is not obvious from the

diffraction results why the glide structure is more stable than the screw, the DFT results indicate that the glide structure has a greater degree of vdW stabilization, suggesting that the angle 
between the two molecules in the unit cell (comparable to the bulk arrangement) allows for a better induced-dipole/induced-dipole interaction between the conjugated $\pi$ systems of phenazine. The implication is that the total polarizability of the overlayer is maximized by having a nonorthogonal intermolecular arrangement of $\pi$ bonds.

Many molecules, including the closely related bipyridine derivatives bpy and bpe, adopt overlayer structures that approximate planes from their bulk crystal structures. Two bulk polymorphs of phenazine have been observed, alpha [35] and beta [31]. Both polymorphs contain chains of offset phenazine molecules, with $\mathrm{C}-\mathrm{H} \cdots \mathrm{N}$ interactions between neighbouring molecules [31]. This arrangement is illustrated in Figure 6.

[Figure 6 near here]

Surprisingly, the phenazine overlayer adopts a herringbone-like structure unrelated to either of the bulk polymorphs. Within the bulk chains, the H-N distances are $2.89 \AA$ for the alpha polymorph, and $2.71 \AA$ for the beta polymorph. The $\mathrm{C}-\mathrm{H} \cdots \mathrm{N}$ angles are $146^{\circ}$ in the alpha polymorph and $156.5^{\circ}$ and $153.9^{\circ}$ in the beta polymorph.

In the overlayer, for the case where the phenazine molecules are constrained to lie flat, the experimentally determined average $\mathrm{H}-\mathrm{N}$ distance is $2.63 \AA$, with a $\mathrm{C}-\mathrm{H} \cdots \mathrm{N}$ angle of $174.7^{\circ}$. For the tilted glide structure, the average $\mathrm{H}-\mathrm{N}$ distance is $3.02 \AA$ and the $\mathrm{C}-\mathrm{H} \cdots \mathrm{N}$ angle is 142. $9^{\circ}$. However, these values must be treated with some caution because the fitting procedure only considers rotations and translations of a rigid molecule taken from the bulk structure, rather than refining individual atom positions as in conventional three-dimensional crystallography. The experimental bond lengths are somewhat larger than those obtained from the DFT 
calculations, which predict an N-H distance of $2.455 \AA$ and $\mathrm{C}-\mathrm{H} \cdots \mathrm{N}$ angle of $175.7^{\circ}$ for the flat structure, and $2.543 \AA$ and $140.7^{\circ}$ for the glide. These distances compare with the sum of the $\mathrm{H}$ and N vdW radii, $2.75 \AA$.

When the molecules are constrained to lie flat, the experimental $\mathrm{H}-\mathrm{N}$ distances are similar to those in the beta polymorph and slightly less than the sum of the vdW radii. This suggests that a weak H-bond would form between the two atoms. For the tilted structure, the experimental H$\mathrm{N}$ distance is now larger than the sum of the vdW radii. However, this is still broadly consistent with the geometries of weak H-bonds, which are more easily distorted by the crystal environment [36]. The experimental $\mathrm{H}-\mathrm{N}$ distances found in the overlayer of the related molecule bpe are $2.57 \AA$ and $2.65 \AA$. This indicates that H-bonding in the phenazine overlayer is weaker than for bpe, which in turn was proposed to be weaker than the $\mathrm{H}$-bonding in the bpy overlayer [17]. The DFT calculations agree well with the experimental results; the energy of the $\mathrm{H}$-bonds was calculated as $0.22 \mathrm{eV}$ per cell for phenazine, compared to $0.48 \mathrm{eV}$ per cell for bpy. The strength of the vdW interactions is broadly similar for both phenazine and bpy $(0.46 \mathrm{eV}$ and $0.39 \mathrm{eV}$ respectively). Hence, the weaker $\mathrm{H}$-bonding in phenazine leads to a lower total layer energy of $0.68 \mathrm{eV}$, compared to $0.87 \mathrm{eV}$ for bpy. This is reflected in the melting points of the overlayers: the phenazine overlayer melts at $274 \mathrm{~K}$, compared to $414 \mathrm{~K}$ for bpe and $442 \mathrm{~K}$ for bpy at similar coverage.

Interestingly, the herringbone structure adopted by phenazine resembles the overlayers of bpe and bpy. For bpe and bpy it was postulated that the herringbone structures observed were driven principally by the formation of weak $\mathrm{C}-\mathrm{H} \cdots \mathrm{N}$ H-bonds, but that the resulting structures would also accommodate a favourable ordering of the molecular quadrupoles. The fact that phenazine forms a herringbone structure dissimilar to the bulk planes, but with considerably 
weaker $\mathrm{C}-\mathrm{H} \cdots \mathrm{N}$ interactions between molecules, provides some tentative evidence that quadrupolar ordering might be an important driving force in the self-assembly of these molecules in two dimensions.

\section{Conclusions}

The overlayer structure of phenazine has been determined by synchrotron X-ray diffraction and DFT calculations and found to consist of a rectangular unit cell containing two molecules. The molecules are found experimentally, and by DFT, to be tilted by $\sim 15^{\circ}$ with respect to the plane of the layer. The experimentally determined structure indicates that the overlayer is uniaxially commensurate with the graphite substrate in the $a$-direction.

Intriguingly, phenazine adopts a herringbone structure in the overlayer, which is different to the bulk crystal. This structure is similar to both bpy and bpe; however, bpy and bpe have C$\mathrm{H} \cdots \mathrm{N}$ interactions that stabilize the overlayer structure, whereas the $\mathrm{C}-\mathrm{H} \cdots \mathrm{N}$ interaction in the phenazine overlayer is much weaker. This is reflected in the lower melting point of phenazine compared to bpy and bpe.

This study, building on previous work, begins to reveal trends in the structural behaviour of simple pyridine-containing physisorbed layers. In combination with DFT calculations on the layer, this has allowed us to separate the relative contribution to the binding energy from van der Waals and other non-covalent interactions. Typically, the pyridine rings physisorb flat on the surface and adopt a herringbone structure. This motif is stabilised by weak $\mathrm{C}-\mathrm{H} \cdots \mathrm{N}$ hydrogen bonds between the pyridine rings, with a possible contribution from a favourable alignment of the molecular quadrupoles.

Essentially, what determines whether two molecules interact via a particular supramolecular motif to form a co-crystal is the balance between the layer energy of the 
supramolecular network relative to the layer energies of the two separated components. Hence a quantitative understanding of the structure and binding energy of the individual components, such as the phenazine monolayer presented here, is an essential constituent in understanding, and ultimately predicting, whether a supramolecular structure will form. In this context, pyridine moieties are an important class of halogen bond acceptors displaying a variety of structural forms. The information gathered in this study is being used in our on-going investigations of halogen bonded co-crystals in monolayers.

\section{Acknowledgements}

We acknowledge financial support for AB from an EPSRC DTA award from the Department of Chemistry, University of Cambridge; and BP for financial support for CT. We thank Diamond Light Source for access to beamline I11 (EE7761) that contributed to the results presented here. The DFT calculations were performed using the Darwin Supercomputer of the University of Cambridge High Performance Computing Service.

\section{References}

[1] K. Morishige and T. Kato, J. Chem. Phys. 111, 7095-7102 (1999).

[2] K. Morishige and Y. Sakamoto, J. Chem. Phys 103, 2354 (1995).

[3] L. Messe, S. M. Clarke, A. Inaba, T. Arnold, C. C. Dong and R. K. Thomas, Langmuir 18, 4010-4013 (2002).

[4] K. Morishige, Y. Takami and Y. Yokota, Phys. Rev. B: Condens. Matter 48, 8277-8281 (1993).

[5] L. Messe, S. M. Clarke, A. Inaba, C. C. Dong, R. K. Thomas, M. A. Castro and M. Alba, Langmuir 18, 9429-9433 (2002).

[6] C. L. Claypool, F. Faglioni, W. A. G. III, H. B. Gray, N. S. Lewis and R. A. Marcus, J. Phys. Chem. B 101, 5978 (1997).

[7] D. M. Cyr, B. Venkataraman, G. W. Flynn, A. Black and G. Whitesides, J. Phys. Chem. 100, 13737-13759 (1996).

[8] A. K. Bickerstaffe, N. P. Cheah, S. M. Clarke, J. E. Parker, A. Perdigon, L. Messe and A. Inaba, J. Phys. Chem. B 110 (11), 5570-5575 (2006).

[9] M. Hibino, A. Sumi and I. Hatta, Jpn. J. Appl. Phys. 34, 3354-3359 (1995).

[10] M. Hibino, A. Sumi, H. Tsuchiya and I. Hatta, J. Phys. Chem. B 102, 4544-4547 (1998).

[11] E. Kishi, H. Matusda, R. Kuroda, K. Takimoto, A. Yamano, K. Eguchi, K. Hatanaka and T. Nakagiri, Ultramicroscopy 42-44, 1067 (1992). 
[12] R. Kuroda, E. Kishi, A. Yamano, K. Hatanaka, H. Matsuda, K. Eguchi and T. Nakagiri, J. Vac. Sci. Technol., B 9, 1180 (1991).

[13] T. Arnold and S. M. Clarke, Langmuir 24, 3325 (2008).

[14] K. S. Mali, B. Van Averbeke, T. Bhinde, A. Y. Brewer, T. Arnold, R. Lazzaroni, S. M. Clarke and S. De Feyter, ACS Nano 5 (11), 9122-9137 (2011).

[15] S. J. H. Griessl, M. Lackinger, M. Edelwirth, M. Hietschold and W. M. Heckl, Single Mol. 3, 25-31 (2004).

[16] S. M. Clarke, T. Friščić, A. Mandal, C. Sun and J. E. Parker, Mol. Phys. 109 (3), 477 $481(2011)$.

[17] A. Y. Brewer, T. Friscic, G. M. Day, L. M. Overvoorde, J. E. Parker, C. N. Richardson and S. M. Clarke, Mol. Phys. 111 (1), 73-79 (2012).

[18] S. M. Clarke, T. Friscic, A. Mandal, C. Sun and J. E. Parker, Chem. Comm. 47, $2526-$ 2528 (2011).

[19] T. Bhinde, S. M. Clarke, T. K. Phillips, T. Arnold and J. E. Parker, Langmuir 26 (11), 8201-8206 (2010).

[20] S. P. Thompson, J. E. Parker, J. Potter, T. P. Hill, A. Birt, T. M. Cobb, F. Yuan and C. C. Tang, Rev. Sci. Instrum. 80 (7), 075107-075109 (2009).

[21] S. P. Thompson, J. E. Parker, J. Marchal, J. Potter, A. Birt, F. Yuan, R. D. Fearn, A. R. Lennie, S. R. Street and C. C. Tang, Journal of Synchrotron Radiation 18 (4), 637-648 (2011).

[22] S. J. Clark, M. D. Segall, C. J. Pickard, P. J. Hasnip, M. I. J. Probert, K. Refson and M. C. Payne, Zeitschrift für Kristallographie - Crystalline Materials 220 (5-6-2005), 567-570 (2005).

[23] M. Sacchi, S. J. Jenkins, H. Hedgeland, A. P. Jardine and B. J. Hinch, J. Phys. Chem. C 115 (32), 16134-16141 (2011).

[24] M. Sacchi, D. J. Wales and S. J. Jenkins, PCCP 14 (45), 15879-15887 (2012).

[25] J. Klimes and A. Michaelides, J. Chem. Phys. 137 (12), 120901 (2012).

[26] S. Grimme, J. Comput. Chem. 27 (15), 1787-1799 (2006).

[27] A. Tkatchenko and M. Scheffler, Phys. Rev. Lett. 102 (7), 073005 (2009).

[28] J. P. Perdew, K. Burke and M. Ernzerhof, Phys. Rev. Lett. 77 (18), 3865-3868 (1996).

[29] A. Guinier and G. Fournet, Small-angle scattering of X-rays. (Wiley, New York, 1955).

[30] N. L. Allinger and G. A. Youngdale, J. Am. Chem. Soc. 84 (6), 1020-1026 (1962).

[31] W. Jankowski and M. Gdaniec, Acta Crystallogr. Sect. C: Cryst. Struct. Commun. 58 (3), 181-182 (2002).

[32] F. Allen, Acta Crystallogr. Sect. B: Struct. Sci. 42 (5), 515-522 (1986).

[33] I. Bandyopadhyay and S. Manogaran, Journal of Molecular Structure: THEOCHEM 507 (1-3), 217-227 (2000).

[34] H. P. Schildberg and H. J. Lauter, Surf. Sci. 208 (3), 507-532 (1989).

[35] F. H. Herbstein and F. R. L. Schoening, Acta Crystallographica 8 (7), 406-412 (1955).

[36] T. Steiner, Chem. Commun. 0 (8), 727-734 (1997). 


\section{Figure Captions}

Figure 1. Molecular structure of (a) phenazine, (b) 4,4'-bipyridine (bpy) and (c) 1,2-bis(4pyridyl)ethylene (bpe).

Figure 2. (a) Experimental and calculated X-ray diffraction patterns of phenazine on graphite at a coverage of 0.51 equivalent monolayers, recorded between $214-239 \mathrm{~K}$. The experimental data is in black and the calculated pattern is in blue. (b) the overlayer structure corresponding to the calculated pattern in (a) with the unit cell shown in red. (c) Electron density difference maps (isosurface level at $0.005 \mathrm{e} \AA$ A 3 ) for phenazine calculated using DFT+D calculations. The grey lines indicate the $\mathrm{p} 2 \mathrm{gg}$ unit cell. Red regions show increase of electron density relative to the separate molecules and dark blue regions indicates a decrease of electron density relative to the separate molecules. In this figure and in the following carbon atoms are grey, hydrogen atoms white, nitrogen atoms light blue.

Figure 3. The fit to the experimental pattern when the phenazine molecules are tilted out of the

plane by $15^{\circ}$. The experimental data are shown in black, and the calculated pattern in blue.

Figure 4. The orientation of the two phenazine molecules in the 'screw' arrangement (left) and 'glide' arrangement (right). The open circle indicates the half of the molecule tilted up from the surface, and the cross indicates the half of the molecule tilted down.

Figure 5. Illustration of the electron density difference maps for BPY overlayer (isosurface level at $0.005 \mathrm{e} / \AA$ 3). The grey lines indicate the unit cell, red regions show increase of electron density relative to the separate molecules and dark blue regions indicates a decrease of electron density relative to the separate molecules. The molecular arrangement is based on that determined in Clarke et al. [16].

Figure 6. The arrangement of molecules into chains in bulk phenazine (taken from the beta polymorph; grey $=$ carbon, blue $=$ nitrogen, white $=$ hydrogen $)$. 